\title{
Ambiguities in the Early Stages of Public Sector Enterprise Architecture Implementation: Outlining Complexities of Interoperability
}

\author{
Hannu Larsson \\ Örebro University, Swedish Business School \\ Fakultetsgatan 1, 70182 Örebro, Sweden \\ hannu. larsson@oru.se
}

\begin{abstract}
In recent years the development of eGovernment has increasingly gone from service provision to striving for an interoperable public sector, with Enterprise Architectures being an increasingly popular approach. However, a central issue is the coordination of work, due to differing perceptions among involved actors. This paper provides a deepened understanding of this by addressing the question of how differing interpretations of interoperability benefits affect the coordination in the early stages of implementing a public sector Enterprise Architecture. As a case-study, the interoperability efforts in Swedish eHealth are examined by interviews with key-actors. The theoretical framework is a maturity model with five levels of interoperability issues and benefits. The findings highlight the need to clarify decision-making roles, ambiguities concerning jurisdictions between authorities and that differing perceptions of IT-infrastructure is connected to overall goals. The paper also suggests a re-conceptualization of eGovernment maturity by moving away from sequential models.
\end{abstract}

Keywords: eGovernment, Interoperability, Maturity models, Implementation, Coordination, Complexity.

\section{Introduction}

In recent years the development of eGovernment has increasingly gone from service provision to integration of back office systems and striving for an interoperable public sector [1]. It has been shown that eGovernment implementation projects tend to be overambitious and often fail, with more or less severe consequences. It has been suggested that this is partly due to that these projects focuses too much on IT (Information Technology), but are rather a part of an agenda of policy change [2, 3]. It has also been shown that IT policies in the public sector often rely on the underlying assumption that technology, by itself, will transform public organizations from stovepipes to integrated administrative systems focused on citizens' needs [4], and that emphasizing IT might lead to a waste of resources as organizational learning and collaboration is not prioritized [5]. Also, there is "overconfidence in the ultimate efficacy of interoperating" in eGovernment projects, meaning that ensuring 
technological interoperability does not necessarily lead to a functionally integrated public sector. This is due to a vast amount of organizational and legal issues [1]. In essence there are several issues related to over empathizing technology over social aspects, i.e. considering the whole Information System (IS), encompassing technology as well as human activities in relation to technology[6]. In trying to treat both social and technical aspects of interoperability different approaches have been taken, with Enterprise Architectures (EA) being increasingly popular [5, 7]. The purpose of EA is to work as a framework or method for aligning business processes and IT use, covering organizational as well as technical aspects [8]. However, previous research shows that EA in the public sector to a large extent is immature, and the IT-architecture and business architectures often are not sufficiently integrated [2], and might be treated separated from each other [7]. Hence the actual efforts to implement interoperability do not necessarily match the overall goals.

Another central implementation issue is the coordination of projects, and creating a shared vision of the future between them [9, 10]. Government IS projects are complex since different organizations or groups of actors might perceive goals differently. This might be problematic as goal convergence (and hence interoperability) might not be reached if different actors act upon incongruent perceptions of goals [11]. In designing an IS the architects are involved in a process of "anticipatory decisionmaking", which involves trying to predict future use, and sets the course for future actions [12]. However, the vision of the future use has some degree of interpretative flexibility and is "a contested object of social and material becoming" and not a point in time where change is bound to happen [13]. Thus, a central aspect is how different organizations and actors differing goals, practices and technologies relates to an overall plan and their interpretation of it [14]. An IS does thus not follow a predictable or neutral trajectory, but is rather dependent upon the specific setting in which it is implemented as different actors might question the intended purpose, and reinterpret it in order to suit local needs [15].

Case studies on the focuses, purposes (perceived benefits) and limitations (issues) to interoperation and integration in eGovernment projects has been suggested as relevant topics for further research [1]. Also, how eGovernment strategies influence interoperability work [5] as well as how motivations are aligned and what the expected outcomes are in large-scale eGovernment projects has been highlighted [16]. This paper addresses the question: How does differing interpretations of interoperability benefits affect the coordination in the early stages of implementing a public Enterprise Architecture? This issue is addressed by means of a case study on the Swedish strategy for eHealth which aims to implement a national EA in the healthcare sector. The case focuses on national and regional collaboration and coordination efforts. As a theoretical framework a maturity model for eGovernment interoperability is used.

\section{Theoretical Framework}

In order to categorize the findings there is a need to apply a theoretical framework to classify which kind of benefits and issues that is treated during implementation. The eGep (eGovernment Economics Project) model provides a framework for achieving a comprehensive theory-based measurement of eGovernment [17] and is used as it has 
been proposed as being suitable for developing a deepened theoretical understanding of eGovernment [18]. The eGep has three value drivers; 1) Financial \& organizational (cashable gains, employee empowerment, improved IT architecture) 2) Political (transparency, accountability, participation) and 3) Constituency (reduced administrative burden, inclusive services, user value) [17]. As the model has a strong emphasis on measurability (e.g. number of services, number of service users etc.) it risks losing in-depth understanding of complex issues [18]. Thus, in this paper only the value dimensions (and not the measurement tools) are used, as the paper focuses on understanding rather than measuring eGovernment. The model has also been criticized for potentially lacking the ability to determine actual progress [18]. Hence, there is a need to combine the model with another model which can assess progress. For this purpose Gottschalk's model for eGovernment interoperability maturity levels is used. It is suitable as it focuses on internal processes of interoperability, instead of more general stage-models that focus on service delivery and/or democracy which is a wider scope then would be useful in this paper. The framework consists of five interoperability levels; 1) Computer - semantic and technical issues, 2) Process linking of work processes and information exchange, 3) Knowledge - IT-enabled knowledge sharing and cooperation among employees from different organizations, 4) Value - combining processes and knowledge sharing to create value by changing practice, and 5) Goal - Ensuring that no conflicting goals exist between the cooperating organizations. The framework is to be used as guidance for research as well as for organizations to be to be able to assess which level they are on and systematically plan development, as organizations reach higher interoperability levels over time. Gottschalk argues that the highest theoretical level of interoperability is not necessarily the most suitable for an organization, as transaction costs might be unnecessarily high if working to achieve a not needed level of interoperability. Gottschalk also argues that further research on variables to be included in the model, and suggests looking into the role of management, legal issues, organizational culture and benefits, as well as the role of technology at each stage [19]. Hence these five aspects are included in order to highlight different kinds of issues. The value drivers from the eGep model represent the perceived benefits.

\section{Method}

As the purpose of the study was to get a deepened understanding of EA implementation, an interpretative case study approach was suitable [20]. Interviews were chosen as the main method for data collection as the involved organizations in the case study are heterogeneous, hence flexibility and scoping was needed [21]. The data material consists of 21 semi-structured interviews. The interviews were divided into two main parts; national actors and regional actors and were performed between August and December 2010. The first four interviewees were selected as they are key actors in the national level work with the EA for the healthcare sector, representing the major organizations involved in the EA-program; One project leader of a keyproject, the head of the national Municipal Coordination Group (MCG), the head of the overall organization of eHealth work and one member of the architecture group in Center for eHealth in Sweden (CeHiS).These interviews were performed to gain a deeper understanding of the plans for implementation, getting access to informants at 
the regional level, and refining the interview guide for the forthcoming interviews. Interviewees for the second stage were selected by recommendations from the national level actors. These were nine municipality coordinators (members of the MCG) and eight County Council actors. Their roles varied and the set included ITprofessionals as well as CIOs. One of the County Council actors was also employed by the national architecture group. These interviews focused on how different actors worked with the strategy on a regional level, perceived benefits, issues and enablers as well as perceptions of future work with interoperability in healthcare.

All interviews were analyzed by identifying relevant statements concerning perceived benefits of interoperability or issues in coordination. Statements concerning similar issues or benefits were grouped into themes and placed in a matrix (Table 1 in the results section). The seven benefits and issues (found on the y-axis) are; The three values from the eGep model (Political-, Constituency- and Financial \& Organizational benefits), which correspond to Gottschalk's suggestion to include benefits. The four issues are Gottschalks other suggested variables (found on the X-axis); Management issues, Organizational culture issues, Legal issues and Technology issues.

An example showing how analysis was done: One municipal coordinator stated that "the central project has decided that we shall use a specific type of eCards which are to be used for healthcare, but it is not suitable for us as we deal with more than just healthcare...". This was categorized as a relating to the computer level (concerning technology) and being a management issue of mapping out which architectural requirements and prerequisites that exist in the involved organizations.

When all themes had been mapped into the matrix an overview of the perceived benefits and issues in the case had been created. These were then analyzed in relation to each other, in order to determine which themes that were related. This led to the themes from the various levels of the model being integrated into three overall themes; Unclear structures and roles for decision-making concerning IT, Ambiguities concerning legal foundations and jurisdictions, and Problems and enablers with following a set path. These overall themes are used to discuss the findings in context, as the perceived benefits and issues reach across several levels of interoperability

\section{Case Description - eHealth in Sweden}

Swedish healthcare is distributed among several care providers. The 290 municipalities are responsible for social services and home care, but also for a vast amount of other services such as schools and waste disposal. Healthcare thus has limited resources and focus. The 20 County Councils are responsible for hospitals and medical care, and almost exclusively focus on healthcare. Healthcare is also handled by private care providers although to a lesser extent.

In 2006 the National strategy for eHealth was released, setting the ground for an EA program. The strategy aims to improve healthcare by means of interoperability, which is seen as a prerequisite for other quality drivers such as allowing patients a choice of provider and efficiency by allowing different providers to be able to hook up on standardized systems. The strategy initially focused on IT and hospital care, as it was mainly driven by an interest group for county councils. In 2010 a new Strategy was released, where the social services care (a municipal responsibility) was also included. A predominant change was also that the focus had now been turned towards 
practice and benefits rather than IT. Since the launch of the initial strategy municipalities have taken a more active position in the program. The program is however funded by county councils and thus municipalities are included to a lesser extent in the development. Neither have all municipalities accepted the strategy, which all county councils have. Under the organization CeHiS there is the previously mentioned MCG which serves as a forum for representatives for all regions (of 10-40 municipalities) to come together and give $\mathrm{CeHiS}$ insight into municipal perspective, as well as bringing knowledge of the national situation back to the municipalities, in order to strive for a more holistic view of the entire program. Henceforth the reader should keep in mind that program refers to the EA program while project refers to smaller parts of it.

\section{Results}

In this section the results are analyzed using the matrix (Table 1) in order to highlight the perceived benefits and issues in achieving these in the implementation of the program. The overall themes (T1, T2 and T3) concern several interoperability levels as well as different types of benefits and issues, and are plotted out in Table 1. Theme 2 covers most of the benefits, while the other themes focus more on issues.

Table 1. The three themes of interoperability benefits and issues plotted out

\begin{tabular}{|l|c|c|c|c|c|}
\hline & Computer & Process & Knowledge & $\begin{array}{c}\text { Value } \\
\text { creation }\end{array}$ & Goal \\
\hline Political benefits & & & & & $\mathrm{T} 2$ \\
\hline Constituency benefits & & $\mathrm{T} 2$ & $\mathrm{~T} 2$ & & $\mathrm{~T} 2$ \\
\hline $\begin{array}{l}\text { Financial \& } \\
\text { Organizational benefits }\end{array}$ & $\mathrm{T} 3$ & $\mathrm{~T} 2$ & $\mathrm{~T} 2$ & $\mathrm{~T} 3$ & $\mathrm{~T} 1, \mathrm{~T} 3$ \\
\hline Management issues & $\mathrm{T} 1, \mathrm{~T} 2, \mathrm{~T} 3$ & & & $\mathrm{~T} 2$ & $\mathrm{~T} 2, \mathrm{~T} 3$ \\
\hline $\begin{array}{l}\text { Organizational culture } \\
\text { issues }\end{array}$ & $\mathrm{T} 1$ & & & & $\mathrm{~T} 1, \mathrm{~T} 3$ \\
\hline Legal issues & $\mathrm{T} 2$ & $\mathrm{~T} 2$ & & $\mathrm{~T} 2$ & $\mathrm{~T} 2$ \\
\hline Technical issues & $\mathrm{T} 1, \mathrm{~T} 3$ & & & & $\mathrm{~T} 1, \mathrm{~T} 3$ \\
\hline
\end{tabular}

\subsection{Theme 1 - Unclear Structures and Roles for IT Decision-Making}

The central issue in this theme concerns where decision making for IT-projects should take place. In the interviews three central questions were raised concerning municipal decision-making; mass, mandate and lack of IT-competence. First, as there are 290 municipalities it is not possible to "sit down in a room together" on a national level. This is also an issue raised by several actors on a regional level, as 10-40 municipalities are also hard to get together. Hence, it is problematic to establish forums for discussion (a computer level management issue), which is dealt with in different ways in different regions. Some regions have joint steering groups with municipal representatives and county council representatives where they discuss eHealth issues together, however not necessarily making decisions. Other regions have more informal cooperation, mainly focusing on informing each other on what is 
going on. In these regions the issues are treated in different groupings with unclear boundaries regarding which issues that are treated where. In these situations the regional municipality coordinator has the task of trying to keep different groupings up to date as well as being informed about what is going on in these. This is experienced as a hard task due to lack of formalized structure for discussions on eHealth issues.

Concerning mandate there are issues regarding how decisions can be taken as well as who has the responsibility to take them. In some steering groups there are municipal representatives with the mandate to make decisions concerning eHealth for the region, by being appointed by other municipalities to do so. More often however the discussions in different groupings can only result in suggestions to the municipalities, leaving them with the option to just say no to decisions that does not suit them. This is raised by several actors. One states that it is about "finding a suitable level of cooperation", not making it too risky or costly for smaller municipalities to accept recommendations. Some of the county council and municipal actors note that decision-making in municipalities tend to be slow and has to run through different levels in order to be approved. Some argue that the slowness is partly due to a lack of IT-competence, especially in smaller municipalities.

Lack of competence on IT (a computer level organizational culture issue) might also result in ambiguities concerning decision-making as IT-projects are traditionally perceived as belonging to IT-departments (a goal level organizational culture issue). Often, municipal politicians do not want to take responsibility for issues concerning what they perceive to be an IT-project, arguing that it belongs to the civil servants in the IT-departments and social services. The problem is that in several of the municipalities it is not clear who should make a decision concerning eCards or who has the competence to deal with requirements for the municipality as a whole and not just for healthcare or by the IT-department (a computer level management issue). From several municipal coordinators' perspective decisions on technologies to procure should be taken with regards to the full responsibilities that municipalities have outside of healthcare, which in their perception needs clearer structures and political support. This conflict concerns the perceived purpose of establishing an ITinfrastructure (a goal level technology issue) which is connected to a financial benefit on the goal level, concerning reduced costs for sharing IT-infrastructure.

\subsection{Theme 2 - Ambiguities Concerning Legal Foundations and Jurisdictions}

The central issue of this theme concerns uncertainties of what was legal to do, and who actually had a say in this. One perceived benefit of working with interoperability is that patient safety will be improved (a value-level constituency benefit) by being able to access relevant information about patients who give their consent. A new law (the Patient Data Act) was introduced in 2008 in order to allow information sharing. However, at a later stage obstacles to this appeared. The (Swedish) Data Inspection Board (DIB) criticized how the law was put in practice as they argued that, during a test run of a key-project, the procedures to ensure patient privacy had not been sufficiently considered, with specific regards to patients who due to mental impairments could not give or deny consent. It was argued that there was no legal ground for medical staff to take part of information regarding these patients as it would be a breach of integrity. Thus, several actors perceive that a central value creation of using eHealth applications was inhibited for a large patient group as the 
constituency benefits of faster information provision and more reliable information (on the process and knowledge level respectively, which also concerns financial benefits on the same levels) were lost due to legal obstacles. Hence, the overall perceived political benefit of patient participation (on the goal level) contained a conflict regarding the legal issues of ensuring data privacy and ensuring patient safety (which concern the process and value level respectively). As an effect of the unexpected interpretation of the law the DIB established itself as a central actor concerning interpretation of laws. In 2010 the DIB urged all organizations implementing the program to be cautious regarding privacy. In several interviews this statement was raised as a central concern for projects under the program, as the DIB does not provide legal advice beforehand. Hence, organizations wanting to implement new projects for which there is no template (such as several projects under the program) are told that they should implement and then the DIB will assess whether or not it lives up to legal requirements. This is experienced as an issue of assuring legal foundations for decision-making (on a computer level) as to avoid forcing local organizations to take high risk decisions.

Being able to do what is best for the patient is expressed as the central constituency benefits (on a goal level) in striving for interoperability. While the Patient Data Act opened up for the county councils sending patient information to municipalities it is not yet legal for municipalities to send such information to the county councils. Hence the program has started to implement an agenda which has requirements that go outside the programs jurisdiction to change (a goal level management issue). Some actors express a concern that it is "not even legal yet" to share information from municipality to county council. Others emphasize that although it is not yet legal laws will "probably be changed". Essentially these different beliefs are about discussing what is thought to be achievable (a value creation level management issue). Some actors argue that as the Patient Data Act has replaced the earlier law it might be possible to change other laws as well in order for the program to function as planned. However, due to the unexpected turn of events with the Patient Data Act, other actors remain skeptical to the possibility of changing other laws. This is a legal issue concerning a conflict between either enabling cross-organizational data sharing by changing laws or adapting the program to existing laws (both on the computer level).

Several of the actors raised issues concerning laws and procurement. The central point is that there is a lack of directives on how to handle joint procurement of services as well as whether different regional organizations could provide services to each other without having to go through a full procurement process. In several regions it was intended that the county councils, being more technically developed, would provide eCards to the municipalities. It was however unclear whether they could do this or not. These uncertainties concerning legal foundations of how to go about implementation costs a lot of time and effort in the regions. This is a problem as several municipalities do not have funding to set up the administrative apparatus for the technological solutions (such as eCards and staff registries) on their own. Thus, several municipalities wish to buy these services from the county council, although are not sure whether they are allowed to do this which is experienced as a legal issue concerning barriers to cooperation in procurement (on the computer level) and a lack of national coordination (a computer level management issue). Several actors experience problems concerning which authority they should listen to and wish for 
greater coordination from a national level, with clearer guidelines to act upon. This is because different authorities might give contradictory statements. One municipal coordinator states that "we get different directives from different directions". For instance it is not clear to the regions under which authorities' jurisdiction certain questions lie, such as how procurement is allowed to be performed. Hence several actors highlighted the need to resolve high-level differences in interpretations of laws (a goal level legal issue). Similarly there is a perceived need to clarify the requirements for technologies in the program. Several actors empathize the perceived need for clear directives (a management issue on the computer level). CeHiS is currently trying to establish itself as a central actor in the Swedish eGovernment setting, which is experienced as problematic by $\mathrm{CeHiS}$ as well as among the regional actors. It is clearly problematic to negotiate with the multitude of other authorities that has a say in Swedish eGovernment and has different perceptions and interpretations, for instance regarding laws. Another example is that there are different opinions concerning standards for eCards, as some argue for the use of digital ID-cards that are handled by banks, while the eGovernment delegation argues for use of more open standards, allowing multiple solutions. This is experienced as problematic in relation to the specific standard for eCards that is already procured for eHealth, which is currently being implemented in several regions.

\subsection{Theme 3 - Problems and Enablers with Following a Set Path}

The central issue in this theme concerns difficulties with new perspectives emerging in the program. As municipalities only recently have started to be involved in the project on a national level and a central issue relates to differing mission scopes of the organizations (a goal level organizational culture issue). Since the initial strategy was mainly driven from a county council perspective the program is now faced with challenges of meeting up with municipal needs. For instance, staff registers and eCards have already been procured and planned into the program, and it thus becomes problematic that some municipalities are skeptical to whether these technologies are suitable for them. While some municipalities follow the lead of the county councils (as suggested by CeHis), others are skeptical towards adopting these technologies. Relating to the regional decision-making several municipalities perceive the eCards as a great challenge as it incorporates a strategic decision that goes outside of eHealth as it involves wider architectural prerequisites for procurement (a technology issue on the computer level). From their perspective the choice of eCards for municipalities should be useable for all sectors (such as for staff in schools). This is connected to that several of the municipalities perceive that working with interoperability in eHealth can act as leverage to interoperability for other sectors as well which conflicts with other actors perceptions of this (which is both a technology and a management issue on the goal level, as it concerns IT-infrastructure and implementation strategy).

As specific eCards and other technologies planned to be used in eHealth might not meet demands from other municipal sectors the skeptical municipalities are put in a problematic situation. Choosing this eCard might, in their perception, cause eHealth to work as a stovepipe as other practices or communication with other authorities, might not be able to use the same eCards as a means for login and identification. This is perceived as a problem for two main reasons; practicality and cost (which is 
financial \& organizational benefits on the value-creation level as well as on a goal level, concerning efficient use of tax-money). One ideal picture raised by some actors is that healthcare should avoid creating yet another system to log in to, but instead strive for single-sign-on. This is also the purpose of the specific eCard for eHealth, although some municipalities do not perceive that this would allow for single-sign-on for municipal users as they will have to communicate with other organizations then the county councils. It is seen as potentially risky and costly due to differing requirements from other authorities. These requirements are not experienced as technically compatible with the eCards for healthcare, which makes some municipalities hesitant towards adopting these solutions. Other municipalities do perceive the eCard as suitable for other municipal sectors as well, and has thus chosen to implement it, although still potentially requiring other cards for communication with other organizations. Another aspect in this is that several county councils and municipalities perceive the specific eCard as a requirement in the program, while others do not, which relates to the issue of which authority that has the mandate in this question (a goal level management issue). Several actors refer to the eGovernment delegations' statements concerning more open standards for eCards and eID, and are thus not willing to implement the specific eCards. The example of eCards is also a symbol for a wider criticism that municipalities prerequisites and requirements has not been sufficiently taken into consideration in the strategy. This issue is also experienced at a national level and concerns ambiguities of what the financial and organizational benefit of better infrastructure (on the computer level) means.

Some of the projects planned to make up the technical infrastructure for eHealth, such as a the specific types of eCards, one of the architects at CeHiS argue, are not in line with what is now considered to be needed for a proper architecture. This is partly due to that municipal needs have not been fully considered. However it is not considered feasible to change the rules during an ongoing game. The architectural group was created after the strategy was designed, and architectural demands are currently being outlined. This is hence done after several technologies have already been procured, started being implemented and are considered to be central to eHealth by several actors. The other architect interviewed (who was also employed by a county council) argues that several issues experienced at a regional level concerning differing architectural preconditions and needs cannot be treated in work on a national level. He states that "The problems experienced in my region are wider then what I am assigned to work with on a national level. [...] Locally we cooperate with municipalities while it is not a part of my assignment nationally". This is because the architectural unit at CeHiS is employed to work from a county council perspective, and not for municipalities. The overall perceived problem is about the inability to adapt to newly discovered architectural prerequisites and needs (a computer level management issue). At the time of writing $\mathrm{CeHiS}$ is in dialogue with other authorities, as well as internally, concerning standards and alternatives for infrastructure.

\section{Conclusions}

In this paper the question raised was how differing interpretations of interoperability benefits affect the coordination in the early stages public sector EA implementation. 
An eGov maturity model was used as the theoretical framework, which clarified how several levels interact. The findings are summarized in four conclusions;

1. In local and regional EA implementation, interpretations of the purpose and scope of a program, and what implications it might have on other parts of eGovernment, can differ. This can lead to ambiguities concerning where decisions should be taken, making coordination hard. EA programs do not only need to acknowledge organizational issues as well as IT, but also needs to highlight the relation to programs in other parts of the public sector, which might overlap.

2. Conflicts are intrinsic to eGovernment programs and relations between national authorities need to be taken into consideration, as unclear jurisdictions might cause confusion among local and regional actors. Hence, being up-front about conflicts, and opening up for discussions on how to deal with them, is vital for coordination.

3. If new organizations get involved in a program, differing goals of implementing a program influence the requirements on IT-infrastructure. Hence, EA programs need to maintain enough flexibility so as to be able to adapt.

4. Using maturity models which perceives development of interoperability as sequential creates an over-simplified picture of implementation. This delimits scientific usefulness, as different "levels" do not follow sequentially. Instead a reconceptualization of maturity models should be discussed. These models have also been criticized by others for being deterministic and simplified [22], which is strengthened by the findings in this paper, as each "stages" cannot be treated separately.

The paper contributes to previous research by providing a deepened understanding of how differing motivations and perceived purposes of interoperability makes alignment problematic. With this paper I hope to raise further discussions concerning these complexities. Further research can address eGovernment evolution with a longitudinal approach, as this case study only covers a limited period of time. Forthcoming research efforts can also elaborate on how to conceptualize eGovernment evolution in ways that incorporates complexity and ambiguities, and does not reduce evolution to something that is assumed to happen in a straight line or through specific stages.

\section{References}

1. Scholl, H., Klischewski, R.: E-Government Integration and Interoperability: Framing the Research Agenda. International Journal of Public Administration 30, 889-920 (2007)

2. Hjort-Madsen, K.: Architecting Government: Underastanding Enterprise Architecture adoption in the public sector. It-University of Copenhagen, Copenhagen (2009)

3. BCS: The challenges of complex IT projects. The report of a working group from The Royal Academy of Engineering The British Computer Society, London (2004)

4. Bekkers, V., Homburg, V.: The Myths of E-Government: Looking Beyond the Assumptions of a New and Better Government. Information Society 23, 373-373 (2007)

5. Klischewski, R., Abubakr, R.: Can e-Government Adopters Benefit from a Technology-First Approach? The Case of Egypt Embarking on Service-Oriented Architecture. 2010 43rd Hawaii International Conference on System Sciences 1-10 (2010)

6. Bacon, J., Fitzgerald, B.: A Systemic Framework for the Field of Information Systems. Database for Advances in Information Systems 32, 46-67 (2009) 
7. Seppanen, V., Heikkila, J., Liimatainen, K.: Key Issues in EA-Implementation: Case Study of Two Finnish Government Agencies. 2009 IEEE Conference on Commerce and Enterprise Computing 114-120 (2009)

8. Guijarro, L.: Semantic interoperability in eGovernment initiatives. Computer Standards \& Interfaces 31, 174-181 (2009)

9. Ebrahim, Z., Irani, Z.: E-government adoption: architecture and barriers. Business Process Management Journal 11, 589-611 (2005)

10.Weerakkody, V., Janssen, M., Hjort-Madsen, K.: Integration and Enterprise Architecture Challenges in E-Government: A European Perspective. International Journal of Cases on Electronic Commerce 3, 13-35 (2007)

11.Shin, D.H.: Convergence and divergence: Policy making about the convergence of technology in Korea. Government Information Quarterly 27, 147-160 (2010)

12.Bell, P., Dean, G., Gottschalk, P.: Information management in law enforcement: The case of police intelligence strategy implementation. International Journal of Information Management 30, 343-343 (2010)

13.Moore, K., Griffiths, M., Richardson, H., Adam, A.: Gendered Futures? Women, the ICT Workplace and Stories of the Future. Gender, Work and Organization 15, 523-542 (2008)

14.Cordella, A., Iannacci, F.: Information systems in the public sector: The e-Government enactment framework. Journal of Strategic Information Systems 19, 52-66 (2010)

15.Elbanna, A., R.: Strategic systems implementation: diffusion through drift. Journal of Information Technology 23, 89-96 (2008)

16.Fedorowicz, J., Gelinas, U.J., Gogan, J.L., Williams, C.B.: Strategic alignment of participant motivations in e-government collaborations: The Internet Payment Platform pilot. Government Information Quarterly 26, 51-59 (2009)

17.Codagnone, C., Boccardelli, P.: eGovernment Economics Project (eGEP) Measurement Framework - Final Version. eGovernment Unit, DG Information Society and Media, European Commission (2006)

18.Grönlund, Å.: Ten years of eGovernment: the end of history and a new beginning. In: Wimmer, M.A., Chappelet, J.-L., Janssen, M., Scholl, H.J. (eds.) 9th IFIP WG 8.5 International Conference on Electronic Government, vol. Proceedings, pp. 13-24. Springer, Lausanne, Switzerland (2010)

19.Gottschalk, P.: Maturity levels for interoperability in digital government. Government Information Quarterly 26, 75-81 (2009)

20.Walsham, G.: Interpretive case studies in IS research: nature and method. European Journal of Information Systems 4, 74-81 (1995)

21.Myers, M.D., Newman, M.: The qualitative interview in IS research: Examining the craft. Information and Organization 17, 2-26 (2007)

22.Coursey, D., Norris, D.F.: Models of E-Government: Are They Correct? An Empirical Assessment. Public Administration Review 68, 523-536 (2008) 